\title{
A CHARACTERIZATION OF LOCALLY GONNEGTED UNICOHERENT CONTINUA
}

PHILIP BACON

(Received 21 September 1966; revised 12 September 1967)

\section{Introduction}

If $\varepsilon>0$, a subset $M$ of a metric space is said to be $\varepsilon$-connected if for each pair $p, q \in M$ there is a finite sequence $a_{0}, \cdots, a_{n}$ such that each $a_{i} \in M, a_{0}=p, a_{n}=q$ and the distance from $a_{i-1}$ to $a_{i}$ is less than $\varepsilon$ whenever $0<i \leqq n$. It is known [1, p. 117, Satz 1$]$ that a compact metric space is connected if and only if for each $\varepsilon>0$ it is $\varepsilon$-connected. We present here a proof of an analogous characterization of locally connected unicoherent compacta.

THEOREM. A connected compact metric space $X$ is unicoherent and locally connected if and only if for each $\varepsilon>0$ there is a $\delta>0$ such that if each of $H$ and $K$ is a closed $\delta$-connected set and $H \cup K=X$ then $H \cap K$ is $\varepsilon$-connected.

The above theorem may be regarded as the principal result of the present paper; a nonmetric variant of it is also proved.

\section{Separation spaces}

According to A. D. Wallace [3], a separation space is a system consisting of a set $X$ and a relation | on the subsets of $X$ obeying these axioms.

(1) $\emptyset \mid X$.

(2) If each of $A$ and $B$ is a subset of $X$ and $A \mid B$ then $B \mid A$.

(3) If each of $A$ and $B$ is a subset of $X$ and $A \mid B$ then $A \cap B=\emptyset$.

(4) If each of $A$ and $B$ is a subset of $X, A_{1}$ is a subset of $A$ and $A \mid B$, then $A_{1} \mid B$.

(5) If each of $A, B$ and $C$ is a subset of $X$ and $A \mid B$ and $A \mid C$ then $A \mid(B \cup C)$.

The symbols ' $A \mid B$ ' are rendered verbally as ' $A$ and $B$ are separated' or ' $A$ is separated from $B$ '. The usual topological concept of separation obeys the separation space axioms. Two additional concepts obeying the axioms are used in this paper. The definitions and theorems for separation 
spaces which we will need are as follows. Proofs of the theorems are in [3]. For the remainder of this section assume that $X$ is a separation space.

Definition. A subset $M$ of $X$ is said to be connected if $M$ is not the union of two nonempty separated sets.

(2.1) If $p \in X$ and $\boldsymbol{G}$ is a collection of connected sets each containing $p$ then $\cup \boldsymbol{G}$ is connected.

Definition. If $M \subset X, C$ is said to be a component of $M$ if $C$ is a connected subset of $M$ that is not a proper subset of any connected subset of $M$.

(2.2) If $M \subset X$, each point of $M$ is in a component of $M$ and no two components of $M$ intersect.

(2.3) If $A$ is separated from $B$ and if $C$ is a connected subset of $A \cup B$ then $C \subset A$ or $C \subset B$.

(2.4) If $K$ is a connected subset of the connected set $M$ and $W$ is a component of $M-K$ then $M-W$ is connected.

\section{A separation concept defined by an open cover of a topological space}

Standing conventions. $X$ is a topological space. $\boldsymbol{E}$ is an open cover of $X$.

Definition. The statement that $A$ is $E$-separated from $B$ means that each of $A$ and $B$ is a subset of $X$ and no member of $E$ intersects both $A$ and $B$.

(3.1) The E-separation concept obeys the axioms for a separation space.

Proofs of the theorems in this section are easily devised and are omitted. In the sequel the words 'connected', 'separated' and 'component' refer to the usual topological separation concept. When the $E$-separation concept is used we shall refer to ' $\boldsymbol{E}$-connectedness' and ' $\boldsymbol{E}$-components'.

(3.2) Each element of $\boldsymbol{E}$ is $\boldsymbol{E}$-connected.

(3.3) Suppose $\boldsymbol{H}$ and $\boldsymbol{G}$ are collections of subsets of $X$ such that if $A \in \boldsymbol{H}$ and $B \in \boldsymbol{G}$ then $A$ is $\boldsymbol{E}$-separated from $B$. Then $\cup \boldsymbol{H}$ and $\cup \boldsymbol{G}$ are E-separated.

(3.4) If $A, B \subset X$, then $A$ is E-separated from $B$ if and only if $\mathrm{Cl} A$ is $\boldsymbol{E}$-separated from $\mathrm{Cl} B$.

(3.5) $A$ subset $A$ of $X$ is $\boldsymbol{E}$-connected if and only if $\mathrm{Cl} A$ is $\boldsymbol{E}$-connected. 
(3.6) If $M \subset X$ and $\boldsymbol{G}$ is a collection each member of which is an $\boldsymbol{E}$ component of $M$ then $\cup \boldsymbol{G}$ is both open and closed in $M$.

(3.7) If $M \subset X$ and $W$ is an E-component of $X-M$ then the boundary of $W$ is a subset of the boundary of $M$.

(3.8) If $A$ and $B$ are E-separated, $A$ and $B$ are separated.

(3.9) If a subset $M$ of $X$ is connected, $M$ is E-connected.

(3.10) If $M \subset X$, each component of $M$ is a subset of an $\boldsymbol{E}$-component of $M$.

DEFINITION. An E-chain from $p$ to $q$ is a finite sequence $B_{0}, \cdots, B_{n}$ such that each $B_{i}$ is in $E, p \in B_{0}, q \in B_{n}$ and $B_{j-1} \cap B_{j} \neq \phi$ whenever $j \in\{1, \cdots, n\}$.

(3.11) If $p, q \in M \subset X, p$ and $q$ belong to the same E-component of $M$ if and only if there is an E-chain $B_{0}, \cdots, B_{n}$ from $p$ to $q$ such that $B_{j-1} \cap B_{j} \cap M \neq \emptyset$ whenever $j \in\{1, \cdots, n\}$.

Notation. If $A \subset X$, define

$$
\operatorname{St}(A, \boldsymbol{E})=\cup\{U \in \boldsymbol{E}: U \cap A \neq \emptyset\} .
$$

(3.12) If each member of $\boldsymbol{E}$ is connected and $A \subset X$ is $\boldsymbol{E}$-connected then $\mathrm{St}(A, \boldsymbol{E})$ is connected.

(3.13) If $P, R, Z \subset X, Z \neq \emptyset$, and no E-component of $Z$ intersects both $P$ and $R$ then $Z$ is the union of two $E$-separated sets, one not intersecting $P$ and the ovher noc intersecting $R$.

For theorems (3.14), (3.15) and (3.16) suppose that $D$ is an open cover of $X$ that refines $\boldsymbol{E}$.

(3.14) If $A$ and $B$ are E-separated then $A$ and $B$ are $D$-separated.

(3.15) If $M$ is D-connected, $M$ is E-connected.

(3.16) If $M \subset X$, each $\boldsymbol{D}$-component of $M$ is a subset of an $\boldsymbol{E}$-component of $M$.

We drop all standing conventions.

(3.17) $A$ closed subset $M$ of a topological space $X$ is connected if and only if, for every open cover $\boldsymbol{E}$ of $X, M$ is $\boldsymbol{E}$-connected.

(3.18) Suppose $X$ is a compact Hausdorff space, $M$ is a closed subset of $X$ and $p$ is in $M$. For each open cover $\boldsymbol{E}$ of $X$, let $M(\boldsymbol{E})$ denote the $\boldsymbol{E}$-component of $M$ that contains $p$. Then

$$
\cap\{M(\boldsymbol{E}): \boldsymbol{E} \text { is an open cover of } X\}
$$

is the component of $M$ that contains $p$. 


\section{Strong unicoherence}

We define four concepts: unicoherence, open-unicoherence, strong unicoherence, and strong open-unicoherence.

A topological space $X$ will be called unicoherent \{open-unicoherent\} if it is nonempty and connected and if for each pair $(H, K)$ such that each of $H$ and $K$ is closed \{open\} and connected and $H \cup K=X$ it is true that $H \cap K$ is connected.

A topological space $X$ will be called strongly unicoherent \{strongly open-unicoherent $\}$ if it is nonempty and connected and if for each open cover $\boldsymbol{E}$ of $X$ there is an open cover $\boldsymbol{D}$ of $X$ such that for each pair $(H, K)$ such that each of $H$ and $K$ is closed \{open\} and $\boldsymbol{D}$-connected and $H \cup K=X$ it is true that $H \cap K$ is $\boldsymbol{E}$-connected.

Because of (3.15) the definition of strong unicoherence is equivalent to the one resulting from imposing the additional requirement that $\boldsymbol{D}$ refine $\boldsymbol{E}$. A similar remark applies to the definition of strong openunicoherence.

(4.1) A strongly unicoherent topological space is unicoherent.

Proof. Suppose $X$ is a connected nonempty space which is not unicoherent. There are closed and connected subsets $H$ and $K$ of $X$ such that $X=H \cup K$ and $H \cap K$ is the union of two nonempty sets $A$ and $B$, separated in the ordinary sense. Define

$$
\boldsymbol{E}=\{X-A, X-B\} .
$$

$E$ is an open cover of $X$. Suppose $\boldsymbol{D}$ is an open cover of $X$. Each of $H$ and $K$ is $\boldsymbol{D}$-connected (3.9). Their intersection is not $\boldsymbol{E}$-connected. $X$ is not strongly unicoherent. $\mathbf{R} \times \mathbb{R}$

The implication in (4.1) cannot be reversed, for the compact subset of

$$
\{(x, \sin (1 / x)): 0<x \leqq 1\} \cup\{(0, y):-1 \leqq y \leqq 1\}
$$

is easily seen to be unicoherent but not locally connected and so, by (4.7) below, cannot be strongly unicoherent.

(4.2) A strongly unicoherent space is strongly open-unicoherent.

Proof. Suppose $X$ is a strongly unicoherent space and $\boldsymbol{E}$ is an open cover of $X$. There is an open cover $\boldsymbol{D}$ of $X$ such that if each of $H$ and $K$ is closed and $\boldsymbol{D}$-connected and $H \cup K=X$ then $H \cap K$ is $\boldsymbol{E}$-connected. Suppose $H$ and $K$ are open and $\boldsymbol{D}$-connected and their union is $X$ but their intersection is the union of nonempty $\boldsymbol{E}$-separated sets $A$ and $B$. By (3.4) $\mathrm{Cl} A$ and $\mathrm{Cl} B$ are $\boldsymbol{E}$-separated. By (3.5) each of $\mathrm{Cl} H$ and $\mathrm{Cl} K$ is $\boldsymbol{D}$-connected. But $\mathrm{Cl} H \cap \mathrm{Cl} K=\mathrm{Cl} A \cup \mathrm{Cl} B$, which is not $E$-connected. This contradiction shows that $H \cap K$ must be $\boldsymbol{E}$-connected. 
That the implication in (4.2) cannot be reversed is shown by the following example of a strongly open-unicoherent space that is neither unicoherent nor open-unicoherent. The space consists of the integers $1,2,3$ and 4 . The topology is

$$
\{\{1,2,3\},\{3,4,1\},\{1\},\{3\}, \emptyset,\{1,2,3,4\}\} .
$$

Definitions. Suppose $X$ is a set and each of $\boldsymbol{D}$ and $\boldsymbol{E}$ is a collection each member of which is a subset of $X$. D is said to star refine $\boldsymbol{E}$ if $\{\mathrm{St}(C, \boldsymbol{D}): C \in \boldsymbol{D}\}$ refines $\boldsymbol{E}$.

A topological space $X$ is said to be fully normal if for each open cover $\boldsymbol{E}$ of $X$ there is an open cover $\boldsymbol{D}$ of $X$ that star refines $\boldsymbol{E}$.

(4.3) A fully normal strongly open-unicoherent topological space is strongly unicoherent.

Proof. Suppose $\boldsymbol{E}$ is an open cover of a fully normal strongly openunicoherent space $X$. Let $\boldsymbol{F}$ be an open cover of $X$ that star refines $\boldsymbol{E}$. Let $\boldsymbol{D}$ be an open cover of $X$ that refines $\boldsymbol{F}$ and is such that, if $H$ and $K$ are open $\boldsymbol{D}$-connected sets whose union is $X$, their intersection is $\boldsymbol{F}$-connected. Suppose $M$ and $N$ are closed $\boldsymbol{D}$-connected sets whose union is $X$ and whose intersection is the union of nonempty $\boldsymbol{E}$-separated sets $A$ and $B$. Since $\boldsymbol{F}$ star refines $\boldsymbol{E}$ and since $A$ and $B$ are $\boldsymbol{E}$-separated, $\operatorname{St}(A, \boldsymbol{F})$ and $\operatorname{St}(B, \boldsymbol{F})$ are $\boldsymbol{F}$-separated. Define

$$
A_{0}=\mathrm{St}(A, \boldsymbol{D}) ; B_{0}=\mathrm{St}(B, \boldsymbol{D}) .
$$

Since $\boldsymbol{D}$ refines $\boldsymbol{F}, A_{0}$ and $B_{0}$ are $\boldsymbol{F}$-separated. Each of $M \cup A_{0} \cup B_{0}$ and $N \cup A_{0} \cup B_{0}$ is an open $\boldsymbol{D}$-connected set, their union is $X$, and their intersection is the union of nonempty $F$-separated sets $A_{\mathbf{0}}$ and $B_{\mathbf{0}}$. Contradiction.

(4.4) A fully normal locally connected open-unicoherent topological, space is strongly unicoherent.

Proof. Suppose $X$ is a fully normal locally connected open-unicoherent space and $\boldsymbol{E}$ is an open cover of $X$. Let $\boldsymbol{F}$ be an open cover of $X$ that star refines $\boldsymbol{E}$. Let $\boldsymbol{D}$ be the collection to which $U$ belongs if and only if $U$ is a component of a member of $\boldsymbol{F} . \boldsymbol{D}$ star refines $\boldsymbol{E}$. Since $X$ is locally connected, $\boldsymbol{D}$ is an open cover of $X$. Suppose each of $H$ and $K$ is closed and $\boldsymbol{D}$-connected and $H \cup K=X$. Define

$$
H_{0}=\operatorname{St}(H, \boldsymbol{D}) ; K_{\mathbf{0}}=\mathrm{St}(K, \boldsymbol{D}) .
$$

By (3.12) each of $H_{0}$ and $K_{0}$ is a connected open set. Since $X$ is openunicoherent, $H_{0} \cap K_{0}$ is nonempty and connected. Suppose $H \cap K$ is the union of nonempty $\boldsymbol{E}$-separated sets $A$ and $B$. Define

$$
A_{\mathbf{0}}=\mathrm{St}(A, \boldsymbol{D}) ; B_{\mathbf{0}}=\mathrm{St}(B, \boldsymbol{D}) \text {. }
$$


Let $p$ be a point of $H_{\mathbf{0}} \cap K_{\mathbf{0}}$. There is a $U$ in $\boldsymbol{D}$ that contains $p$ and intersects $H$, and a $V$ in $\boldsymbol{D}$ that contains $p$ and intersects $K$. $U \cup V$ is a connected set that is the union of the nonempty sets $(U \cup V) \cap H$ and $(U \cup V) \cap K$. By (2.3) their intersection is nonempty, that is,

$$
\emptyset \neq(A \cap U) \cup(A \cap V) \cup(B \cap U) \cup(B \cap V) .
$$

Thus $p$ is in a member of $\boldsymbol{D}$ that intersects one of $A$ and $B$. Therefore $p \in A_{0} \cup B_{0}$ and $H_{0} \cap K_{0} \subset A_{0} \cup B_{0}$. Since $A_{0}$ and $B_{0}$ are open and $H_{0} \cap K_{0}$ is a nonempty connected set that intersects both $A_{0}$ and $B_{0}$ and is contained in their union, $A_{0}$ and $B_{0}$ have a point $t$ in common. St $(\{t\}, \boldsymbol{D})$ intersects both $A$ and $B$. Since $\boldsymbol{D}$ star refines $\boldsymbol{E}$, there is a $W$ in $\boldsymbol{E}$ that contains St $(\{t\}, \boldsymbol{D})$ and therefore intersects both $A$ and $B$. This contradicts the assumption that $A$ and $B$ are $\boldsymbol{E}$-separated. $A$ and $B$ do not exist and $H \cap K$ is $\boldsymbol{E}$-connected.

Definition. If $\boldsymbol{D}$ is an open cover of a topological space $X$, the statement that $M D$-separates $p$ from $q$ means that $M \subset X$ and $X-M$ is the union of two $D$-separated sets, one containing the point $p$ and the other containing the point $q$.

(4.5) If each or $\boldsymbol{E}$ and $\boldsymbol{D}$ is an open cover of the connected nonempty topological space $X$ and $\boldsymbol{D}$ refines $\boldsymbol{E}$ then these are equivalent:

(a) If each of $A$ and $B$ is an open $D$-connected set and $A \cup B=X$ then $A \cap B$ is $\boldsymbol{E}$-connected.

(b) If $M$ and $N$ are disjoint closed sets neither of which $D$-separates $p$ from $q$ then $M \cup N$ does not $\boldsymbol{E}$-separate $p$ from $q$.

Proof. (a) $\Rightarrow$ (b). Let $\boldsymbol{H}$ be the collection of $\boldsymbol{D}$-components of $X-M$, let $\boldsymbol{K}$ be the collection of $\boldsymbol{D}$-components of $X-N$, and let $\boldsymbol{J}$ denote $\boldsymbol{H} \cup \boldsymbol{K}$. By (3.6) $\boldsymbol{J}$ is an open cover of $X$. Call two subcollections $\boldsymbol{F}$ and $\boldsymbol{G}$ of $\boldsymbol{J}$ $S$-separated if $\cup \boldsymbol{F} \cap \cup \boldsymbol{G}=\emptyset$. This definition makes $\boldsymbol{J}$ a separation space. Notice that if a subcollection $\boldsymbol{G}$ of $\boldsymbol{J}$ is $S$-connected then $\cup \boldsymbol{G}$ is $\boldsymbol{D}$-connected and, if two elements of $\boldsymbol{J}$ intersect, one is in $\boldsymbol{H}$, the other is in $\boldsymbol{K}$ and their intersection is a subset of $X-(M \cup N)$.

Suppose $M \cup N E$-separates a point $p$ from some point $q$, that is, $X-(M \cup N)$ is the union of two $E$-separated sets $A$ and $B, A$ containing $p$ and $B$ containing $q$. Suppose also that neither $M$ nor $N \boldsymbol{D}$-separates $p$ from $q$. There is a member $U$ of $\boldsymbol{H}$ containing both $p$ and $q$ and there is a member $V$ of $\boldsymbol{K}$ containing both $p$ and $q$. If $U=V$ then $U$ is a $\boldsymbol{D}$-connected (and $E$-connected) subset of $X-(M \cup N)$ that contains both $p$ and $q$, which contradicts our assumption that $M \cup N E$-separates $p$ and $q$; so $U \neq V$. Let $\boldsymbol{W}$ denote the $S$-component of $\boldsymbol{J}-\{U\}$ that contains $V$. By (3.2) and (2.4) $\boldsymbol{J}-\boldsymbol{W}$ is $S$-connected. Each of $\cup \boldsymbol{W}$ and $\cup(\boldsymbol{J}-\boldsymbol{W})$ is open 
and $\boldsymbol{D}$-connected and their union is $X$. By (a), $\cup \boldsymbol{W} \cap \cup(\boldsymbol{J}-\boldsymbol{W})$ is an $\boldsymbol{E}$-connected subset of $A \cup B$ and is accordingly entirely a subset of one of $A$ and $B$, say $A$. Since $U \in \boldsymbol{W}$ and $V \in \boldsymbol{J}-\boldsymbol{W}, U \cap V \subset A$. So $q \in U \cap V \subset A$, which contradicts $q \in B$.

(b) $\Rightarrow$ (a). Suppose each of $A$ and $B$ is an open $\boldsymbol{D}$-connected set, $A \cup B=X$ and $A \cap B$ is the union of $E$-separated sets one of which contains a point $p$ and the other of which contains a point $q . X-A$ and $X-B$ are disjoint closed sets neither of which $D$-separates $p$ from $q$. By (b), $(X-A) \cup(X-B)=X-(A \cap B)$ does not $E$-separate $p$ from $q$. Contradiction.

Definition. If $X$ is a topological space and $p \in X$, the quasi-component of $X$ that contains $p$ is the common part of all sets containing $p$ that are both open and closed.

It is not hard to show that a topological space is locally connected if and only if each quasicomponent of each open set of the space is open.

(4.6) A regular strongly open-unicoherent space is locally connected.

Proof. Suppose, on the contrary, there is a regular strongly openunicoherent space $X$ that is not locally connected. Then $X$ must contain an open set $V_{1}$ one of whose quasicomponents is not open. Let $p$ be a point of $V_{1}$ that is not in the interior of the quasicomponent $Y$ of $V_{1}$ that contains $p$. Since $X$ is regular, we may choose successively open neighborhoods $V_{2}, V_{3}$, and $V_{4}$ of $p$ such that $\mathrm{Cl} V_{2} \subset V_{1}, \mathrm{Cl} V_{3} \subset V_{2}$, and $\mathrm{Cl} V_{4} \subset V_{3}$. Define

$$
\boldsymbol{E}=\left\{V_{3}, X-\mathrm{Cl} V_{4}\right\}
$$

$\boldsymbol{E}$ is an open cover of $X$. Since $X$ is strongly open-unicoherent, there is an open cover $\boldsymbol{D}$ of $X$ that refines $\boldsymbol{E}$ and is such that if each of $F$ and $G$ is an open $\boldsymbol{D}$-connected set and $F \cup G=X$ then $F \cap G$ is $\boldsymbol{E}$-connected. Let $A$ be an element of $\boldsymbol{D}$ that contains $p$. There is a point $r$ in $\left(A \cap V_{4}\right)-Y$. Since $Y$ is a quasicomponent of $V_{1}$ and $p$ is in $Y$ and $r$ is in $V_{1}-Y, V_{1}$ is the union of disjoint open sets $K_{p}$ and $K_{r}, K_{p}$ containing $p$ and $K_{r}$ containing $r$. Define

$$
H_{p}=K_{p} \cap \mathrm{Cl} V_{2} ; H_{r}=K_{r} \cap \mathrm{Cl} V_{2} .
$$

Since each of $K_{p}$ and $K_{r}$ is closed in $V_{1}$, each of $H_{p}$ and $H_{r}$ is closed. Let $\boldsymbol{C}$ be an open cover of $X$ that refines each of $\boldsymbol{D},\left\{X-H_{p}, X-H_{r}\right\}$ and $\left\{X-\mathrm{Cl} V_{3}, V_{2}\right\}$. Since $X$ is connected, $X$ is $\boldsymbol{C}$-connected (3.9), and there is a $\mathcal{C}$-chain from $p$ to $r(3.11)$. Let $B_{0}, \cdots, B_{n}$ be a $\mathcal{C}$-chain from $p$ to $r$ such that no $\boldsymbol{C}$-chain from $p$ to $r$ has fewer terms that are entirely a subset of $X-\mathrm{Cl} V_{3}$. Since $H_{p}$ and $H_{r}$ are $\mathcal{C}$-separated, no $B_{j}$ intersects both $H_{p}$ and $H_{r}$. Hence there is an integer $i$ and a point $q$ such that $q \in B_{i}-\mathrm{Cl} V_{\mathbf{2}}$. Since $C$ is a refinement of $\left\{X-\mathrm{Cl} V_{3}, V_{2}\right\}, B_{i} \subset X-\mathrm{Cl} V_{3}$. Define 


$$
S_{p}=\bigcup_{j=0}^{i-1} B_{j} ; S_{r}=\bigcup_{j=i+1}^{n} B_{j} .
$$

$S_{p}$ and $S_{r}$ are nonempty since $p \in S_{p}$ and $r \in S_{r}$.

Suppose there is a $\mathcal{C}$-component of $\mathrm{Cl} V_{\mathbf{3}}-V_{\mathbf{4}}$ that intersects both $S_{p}$ and $S_{r}$. By (3.11) there is a $\boldsymbol{C}$-chain $T_{0}, \cdots, T_{m}$ from a point of $S_{p}$ to a point of $S_{r}$ such that each $T_{j}$ intersects $\mathrm{Cl}_{3}$. There is a chain from $p$ to $r$ each term of which is one of $B_{0}, \cdots, B_{i-1}, B_{i+1}, \cdots, B_{n}, T_{0}, \cdots, T_{m}$. Such a chain has fewer terms that are a subset of $X-\mathrm{Cl} V_{3}$ than does $B_{0}, \cdots, B_{n}$. Since this contradicts our choice of $B_{0}, \cdots, B_{n}$, our assumption that there is a $\mathcal{C}$-component of $\mathrm{Cl} V_{3}-V_{4}$ that intersects both $S_{p}$ and $S_{r}$ is not valid.

It follows by (3.13) and (3.6) that $\mathrm{Cl} V_{3}-V_{4}$ is the union of $C$-separated closed sets $M$ and $N, M$ not intersecting $S_{p}$ and $N$ not intersecting $S_{r}$. $M$ does not intersect the $D$-connected set $S_{p} \cup B_{i}$ and $N$ does not intersect the $\boldsymbol{D}$-connected set $\{p\} \cup S_{\boldsymbol{r}} \cup B_{\boldsymbol{i}}$. Thus, neither $M$ nor $N \boldsymbol{D}$-separates $p$ from $q$. This contradicts (4.5).

(4.7) If $X$ is a regular fully normal space, these are equivalent:

(a) $X$ is strongly unicoherent.

(b) $X$ is strongly open-unicoherent.

(c) $X$ is unicoherent and locally connected.

(d) $X$ is open-unicoherent and locally connected.

Proof. That (a) implies (b) follows from (4.2). That (b) implies (c) follows from (4.3), (4.1) and (4.6). The equivalence of (c) and (d) (even in the absence of regularity and full normality) is a result of $A$. H. Stone $[2$, p. 432, Theorem 3]. That (d) implies (a) follows from (4.4).

\section{Strong unicoherence in compact metric spaces}

Throughout this section assume that $X$ is a metric space with the distance from $p$ in $X$ to $q$ in $X$ denoted by $|p-q|$.

Definition. The statement that $A$ is $\varepsilon$-separated from $B$ means that each of $A$ and $B$ is a subset of $X, \varepsilon$ is a positive number, and $|p-q| \geqq \varepsilon$ whenever $p \in A$ and $q \in B$.

(5.1) Suppose $\varepsilon>0$ and $E(\varepsilon)$ is the set of all open subsets of $X$ of diamerer less than $\varepsilon$. Then $A$ is e-separated from $B$ if and only if $A$ is $\boldsymbol{E}(\varepsilon)-$ separated from $B$.

It follows from (3.1) and (5.1) that the $\varepsilon$-separation concept obeys the axioms for a separation space. The definition of connectedness corre- 
sponding to this separation space is easily seen to be equivalent to the definition of $\varepsilon$-connectedness given in section 1 .

(5.2) If $X$ is compact and $\boldsymbol{E}$ is an open cover of $X$, there is an $\varepsilon>0$ such that if $M$ is an $\varepsilon$-connected subset of $X$ then $M$ is $E$-connected.

The reader may prove (5.1) and (5.2) and then use them to show

(5.3) If $X$ is a nonempty compact connected metric space, these are equivalent:

(a) $X$ is strongly unicoherent.

(b) If $\varepsilon>0$, there is a $\delta>0$ such that if each of $A$ and $B$ is a closed $\delta$-connected set and $A \cup B=X$ then $A \cap B$ is e-connected.

The theorem of section 1 follows from (5.3) and (4.7).

6

I am indebted to the referee for the following observation. Call a connected nonempty topological space $X$ semi-strongly $\{0 p e n\}$ unicoherent if for every finite (or, equivalently, two-element) open cover $\boldsymbol{E}$ of $X$ there is an open cover $\boldsymbol{D}$ of $X$ such that if each of $H$ and $K$ is closed \{open\} and $\boldsymbol{D}$-connected and $H \cup K=X$ then $H \cap K$ is $\boldsymbol{E}$-connected. Then the theorems and arguments of this paper go through with strong unicoherence replaced by semi-strong unicoherence and in (4.3) and (4.4) full normality weakened to normality (since finite open covers of normal spaces have finite star refinements). The list of equivalent conditions in (4.7) can be extended to include semi-strong unicoherence and semi-strong openunicoherence.

\section{References}

[1] P. Alexandroff and H. Hopf, Topologie, Chelsea Publishing Company, Bronx, New York, 1965.

[2] A. H. Stone, 'Incidence relations in unicoherent spaces', Trans. Amer. Math. Soc. 65 (1949), 427-447.

[3] A. D. Wallace, 'Separation spaces', Ann. of Math. (2) 42 (1941), 687-697.

University of Florida

Gainesville, Florida 